Proceedings of the Annual Scientific Meeting of the Society held on 27 to 29 July 1967, at Stoke Mandeville Hospital, Aylesbury

\author{
PART II
}

ASSOCIATED INJURIES IN TRAUMATIC PARAPLEGIA

AND TETRAPLEGIA

\title{
THE EARLY MANAGEMENT OF ASSOCIATED INJURIES IN THE PRESENCE OF CO-INCIDENT DAMAGE TO THE SPINAL CORD
}

\author{
By Terence McSweeney, M.Ch.(N.U.I.), M.Ch.(Orth.), F.R.C.S.(Eng.) \\ Spinal Injuries Unit, Oswestry. \\ The Robert Fones and Agnes Hunt Orthopaedic Hospital, Oswestry
}

THE problem is becoming an increasingly important one because of the upward trend in road traffic accidents-the skill and speed with which the severely injured are transported to hospital, and the advances in resuscitation.

Mining, construction and demolition work, mechanised agriculture, heavy industry, war wounds and stab wounds have always accounted for a certain number of cases in which the spinal injury was associated with other injuriesusually to the limbs.

The athletic accident pattern, apart from mountaineering, is seldom one of multiple injuries.

Traffic accidents, by contrast, may be associated with multiple damage in the spine-quite apart from the cord lesion, as well as a kaleidoscope of associated injuries.

Case Report.-In April I963, a 23-year-old man was involved in a road accident. Among other injuries he sustained a severe 'crush injury' of the chest with multiple rib fractures accompanied by a large haemo-pneumothorax. This was associated with an extensive rupture of the left dome of the diaphragm and displacement of some of the abdominal contents into the left side of the chest. There was a gross diastasis of the pelvis with rupture of the prostatic urethra and of the anterior bladder wall. In addition, he was totally paraplegic from the Ioth dorsal segment because of a grossly unstable fracture of the IIth on the I2th dorsal vertebra.

$\mathrm{He}$ is at present employed in a full-time research capacity in one of our older universities. He is sufficiently independent to live on his own.

Management. In the initial assessment of any severely injured patient, once an adequate airway has been established, it is important to make a mental note of the probable blood loss, to check the peripheral pulses and to record the blood pressure (if possible).

A clear account of the circumstances of the accident should alert the mind to the injury pattern and the various possibilities.

Assuming that the level and completeness or otherwise of the cord injury has been established, it is convenient to assess the obvious injuries first-these 
usually involve the limbs. This should take a short time and allow ample time for a consideration of the so-called 'silent areas' - the chest, head and abdomen.

Resuscitation. In cervical injuries, we have been guided for the most part by the work of Cheshire and Coats (I966).

But we find a decreasing indication for tracheostomy except in the presence of pre-existing lung disease, severe associated chest or head injury. This is largely due to the increased experience of the staff, particularly the unit physiotherapists, and attention to the need for bronchial aspiration, gastric suction and the control of body and environmental temperature.

Tracheostomy is seldom necessary in dorsal or lumbar paraplegia, except in the presence of a severe chest injury. When the associated chest injury is less severe, endotracheal intubation will often tide the patient over the first day when the need for tracheostomy can be reassessed.

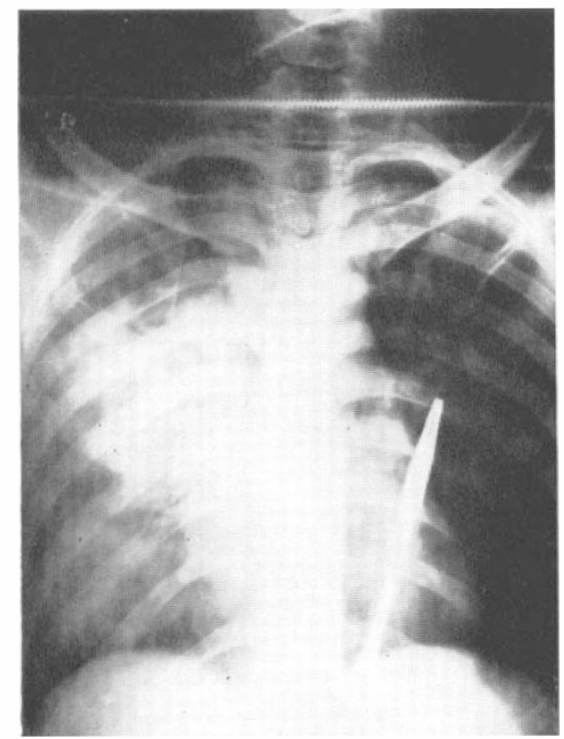

FIG. I

Oedema of lung following intravenous administration of 4 litres of blood in I2 hours. The patient had closed fractures of both femoral shafts in addition to a complete cord lesion at the sixth cervical segment.

Blood Transfusion. There is a dilemma here because of the interference with vasomotor control. 'Rule of thumb' assessment of blood loss, as a result of the associated injuries, may indicate a figure beyond the patient's cardiovascular capabilities. At the present time the estimation of blood loss is an intelligent guess based on the clinician's previous experience of similar injuries. It takes into account the nature, site and number of such injuries, the blood loss through compound wounds and the probable blood loss if surgery is contemplated. 
Frequent estimation of the central venous pressure and of the urinary output help to guard against circulatory overloading.

A familiarity with the work of the late Ruscoe Clarke (I955) and his colleagues at the Birmingham Accident Hospital forms a useful background to the problem of blood loss.

Unfortunately I have no practical experience of the various machines which quickly and accurately measure blood volume. Clearly such estimates frequently repeated would be of inestimable value.

The massive transfusion, so vital in the resuscitation of the young adult with severe multiple injuries and an intact spinal cord, is compromised in the presence of spinal shock. Despite awareness of the loss of vasomotor control in tetraplegics, we have more than once fallen into the error of over-transfusion (fig. I).

More often than not injury to the cervical cord is an isolated one, or associated with head or upper limb injuries where blood loss into the tissues is seldom severe. I suspect that when a cervical cord injury is associated with severe haemorrhage from multiple injuries, the patient is dead on arrival at the hospital.

In dorsal and lumbar paraplegia the risk of over-transfusion is not so great, particularly so when the stage of spinal shock has passed. We should be mindful however that the spinal reflexes controlling peripheral vascular tone take a varying length of time to recover, depending on the site and nature of the spinal cord injury.

The use of fresh packed cells has the obvious advantage of lessening the volume of intravenous fluid required to restore oxygen-carrying capacity. Nevertheless, circulatory overloading and pulmonary oedema are easily produced and their prevention requires the utmost clinical vigilance.

Jugular filling, improvement in capillary tone, the effect on blood pressure, repeated examination of the lung bases and X-rays of the chest are important guides to the rate and amount of transfusion. Frequent revision of the original estimate in the light of clinical response and estimation of the central venous pressure should minimise the risk of over-transfusion. In general it is better to cannulate the upper limbs rather than the lower limbs. Apart from other considerations, this may have some bearing on the later development of deep vein thrombosis. I have no experience of intra-arterial transfusion. Simple measures, such as elevation of the foot end of the bed, should not be overlooked. A readily available clinical estimation of extracellular fluid content would simplify the management of these difficult problems.

Management of Limb Injuries. Soft tissue wounds and compound fractures are thoroughly cleaned, devitalised tissue excised and simple closure undertaken as soon as the patient's general condition will permit.

This is not the time for elaborate skin grafting procedures.

Once gross deformity is corrected, well-padded plaster gutter splints are used to maintain alignment. For single bone injuries, it may be possible to dispense with splints and make use of a pillow and crepe bandage to support the limb.

Uncomplicated dislocations are promptly and gently reduced.

In a paralysed limb, only token immobilisation is required-to remind all concerned that the joint has been dislocated.

Passive movements after reduction of a dislocation, so vital in the uninjured 
limb, if carried out too early or too vigorously may end in joint stiffness (myositis ossificans post traumatica) or redislocation.

In closed fractures, inflatable splints are often useful as a temporary measure, particularly in the unparalysed upper limb of paraplegic patients. There is seldom need for a general anaesthetic at this stage.

Some hours later, with improvement in the patient's general condition, the question of more definite treatment of limb injuries will arise.

In lumbar paraplegia, the patient is usually fit for surgery (if such is thought necessary) within five to six hours of admission to hospital. Dorsal injuries are often the result of traffic accidents and are associated with a high incidence of other injuries. They are seldom fit, except for the most compelling limb surgery, during the first 48 hours.

Cervical injuries usually remain in a precarious state for four to five days. In my experience, the pattern of injury is such that surgery is rarely indicated during the first two to three weeks.

Over and above these considerations, the timing of surgery is largely a question of experience-conditioned not only by the spinal state but the presence of chest, head or abdominal injuries.

It is my policy to treat fractures of the femoral shaft, when an isolated injury, on a Thomas splint. In the presence of multiple injuries or a fracture of the ipsilateral tibia, I frequently employ internal fixation by an intramedullary nail. It is a question of adjusting the treatment of a particular fracture against the whole background of the patient's general and clinical requirements.

In considering closed fractures, it is convenient to recognise-Absolute Indications, Relative Indications and Contra-Indications for surgery.

Absolute Indications. Fractures accompanied by gross pressure on vessels or nerves, or where an attempt at closed reduction may increase the neurovascular hazard, require exploration and reduction.

Such injuries occur near joints or are associated with fracture-dislocations, e.g. supracondylar fractures of the humerus, fracture-dislocations of the shoulder, and tarso-metatarsal region.

Dislocations of the semi-lunar bone and other dislocations around the wrist are sometimes associated with pressure on the median nerve.

Contra-indications. Certain fractures seldom if ever require open reduction provided there is no gross pressure on vessels or nerves. These include:

(a) All diaphyseal (shaft of long bone) fractures in childhood.

(b) Most fractures of the scapula and pelvis.

(c) Fractures of the clavicle.

(d) Fractures of the os calcis other than avulsion injuries.

(e) Most comminuted fractures near the ends of long bones.

Relative Indications. This is the group where opinions will differ. It can be expanded depending on the general condition of the patient, the facilities available, the experience and temperament of the surgeon. The surgeon unaccustomed to the discipline of open reduction should not attempt heroic surgery in these patients.

In the potentially paralysed limb, one must aim for ultimate freedom of joint movement above all else. 
It is not advisable to accept gross angular deformity in an anaesthetic limb because of the difficulty in fitting appliances at a later stage.

Internal fixation of the paralysed limb usually disposes of the need for splintage of any kind. It often allows the paraplegic to sit out of bed earlier-and to commence his rehabilitation programme sooner.

I should emphasise, however, that unless the surgeon is familiar with the operative management of fractures it is a grave error to equate open reduction with perfection.

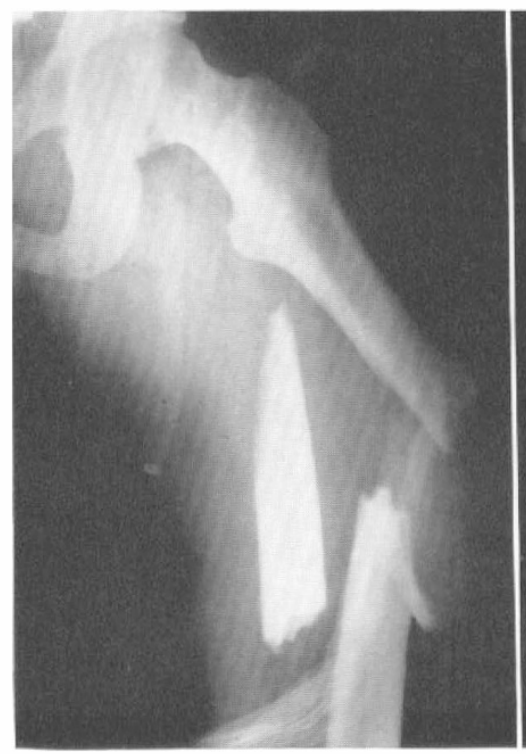

A

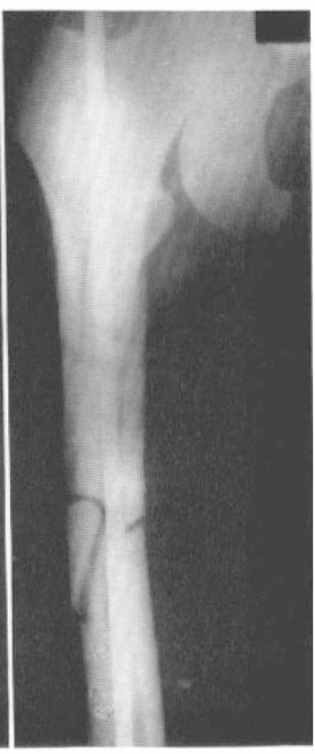

B

FIG. 2

High velocity fracture of femur treated by fixation with intramedullary nail.

For this reason it has been argued that some of these injuries should wait for later corrective surgery. Delayed surgery is in my view seldom satisfactory in terms of joint movement, particularly when the initial injury is an intra-articular one. Furthermore, late correction of angular deformity of the shaft of a long bone, even with planned shortening, may jeopardise the function in the adjacent joint.

If the patient can be made reasonably comfortable on temporary splintage and when circulation and nerve conduction are not in danger, I find good reasons for delaying limb surgery in paraplegics for three to four days. In tetraplegics, the time interval can be longer and the need for surgery is usually less.

A reasonable time should elapse to allow maximum recovery in the autonomic nervous system. Further investigation into this problem and the choice of anaesthetic agent is urgently needed.

With the foregoing thoughts in mind, open reduction should be considered in: 
I. Fractures which are irreducible, often because of the interposition of soft tissues, e.g. high velocity fractures of the femur (fig. 2); certain fractures at the upper end of the humerus where the biceps tendon slips between the head and the shaft; tarso-metatarsal injuries where the tibialis anterior is interposed between the bone ends; separation of the medial malleolus with interposition of the tibialis posterior tendon.

2. Fractures which are inherently and grossly unstable, e.g. shaft of ulna with dislocation of radial head (Monteggia); fracture of radius at junction of middle and lower thirds (Galeazzi); many fractures in which both forearm bones are broken; multiple limb fractures (fig. 3); certain segmental fractures, and fractures of the femoral neck.

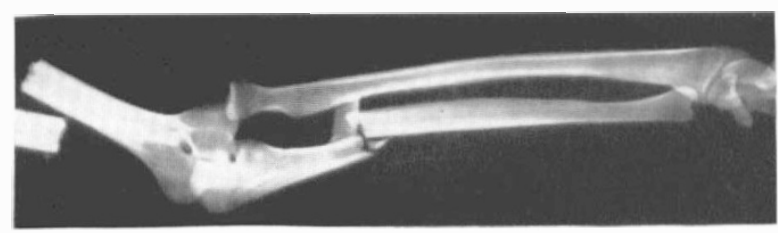

A

FIG. 3

Multiple injuries of upper limb treated by internal fixation.

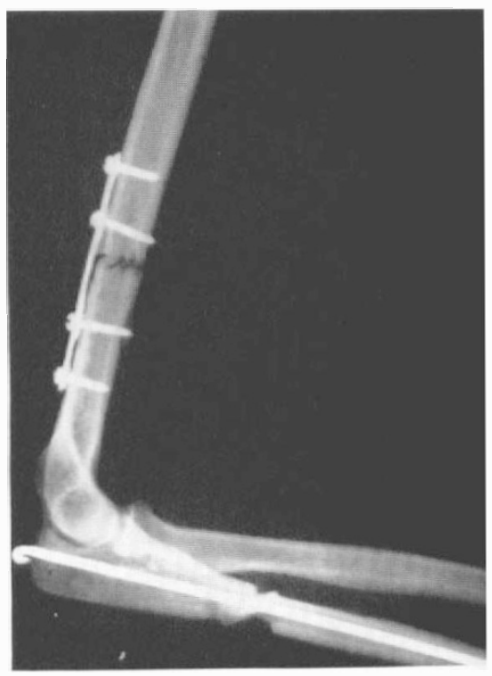

$\mathrm{B}$

3. Fractures where the fragment is so small that it cannot be manipulated or where the fragment is trapped in the joint as a 'loose body'. Examples are: dislocation of elbow, with inclusion of the medial epicondyle in the joint; fracture separation of the capitellar epiphysis; fracture of the radial head with severe tilting.

4. Fractures where the fragment forms an integral part of the joint, e.g. fracture of the postero-superior margin of the acetabulum or associated fracture of the femoral head; bimalleolar fractures of the ankle; fracture of the femoral condyle with separation of the articular surface. os calcis.

5. Certain avulsion injuries, e.g. patella, olecranon, and posterior tubercle of

6. In hopelessly injured limbs, a place for timely amputation should not be forgotten.

In the management of associated injuries to the head, chest and abdomen, surgeons from the appropriate disciplines will speak with much more clarity and authority than I can. Nevertheless, we are all concerned in the total management of the patient and I hope my personal views will not be out of place. 
Head Injuries. It is surprising how the state of consciousness improves with correction of respiratory obstruction.

In the unconscious patient, fractures of the spine in general and of the cervical spine in particular are easily missed. I emphasise the point that if the patient is to have the skull X-rayed, he should have simultaneous X-rays of the neck.

Operation is required only in a minority of head injuries. I recognise three indications for really urgent cranial surgery:

I. Compound fracture of the skull.

2. Expanding lesions within the skull, usually a middle meningeal haemorrhage.

3. Certain fractures across the sinuses.

Abdominal Injuries. The insertion of a fine polythene nasogastric tube is an essential initial measure.

Open wounds of the abdomen associated with spinal injuries are, for the most part, injuries of warfare and the need for exploration is obvious.

Closed injuries of the abdomen accompanied by spinal injuries are, mercifully, rare, even in road traffic accidents: I can only recall three cases. I presume the combination of a cord injury with serious intra-abdominal damage is more often than not too overwhelming for survival.

The fundamental drill of Inspection, Percussion, Palpation and Auscultation may throw little or no light on the problem when the spinal cord is damaged.

In particular, absence of bowel sounds in the first 24 to 48 hours after injury is not an indication for laparotomy. The abdominal rigidity associated with fractures of the lumbar spine is a trap for the unwary.

Local bruising or abrasions of the abdominal wall, together with an accurate history, careful clinical investigation frequently repeated and X-ray studies including the use of contrast media will help in deciding about laparotomy or the need for intrapelvic surgery.

Evidence of haemorrhage and shock over and above what might be expected, or the failure of response to adequate resuscitative measures, should arouse suspicion of an intra-abdominal injury.

It is a mistake to approach the problem in terms of damage to individual organs. As in the non-spinal case-when in serious doubt, explore, for the maximum period of resuscitation is all too short.

Chest Injuries. In dorsal and lumbar lesions, it is usually possible to avoid tracheostomy-utilising temporary endotracheal intubation with intermittent positive pressure anaesthesia for the first 24 to 48 hours. In tetraplegic patients with severe associated chest injuries, tracheostomy is vital.

In all cases adequate humidification and high quality barrier nursing are essential.

Five other basic principles must be remembered:-

(a) The relief of tension pneumothorax by a hollow needle.

(b) Immediate closure of a sucking pneumothorax (open pneumothorax).

(c) Correction of paradoxical respiration due to 'flail chest'.

(d) Frequent bronchoscopic toilet.

(e) Aspiration of the pleural cavity is often advisable and when intercostal 
drainage is necessary, it should be carried out through a wide-bore tube with an under-water seal.

There is no doubt in my mind that our colleagues in Anaesthesia have, to a large extent, overcome these problems by the use of intermittent positive pressure ventilation. So much so, that fixation of a flail chest by mechanical devices is seldom indicated.

I have not touched on mediastinal haemorrhage-injuries to the heart, bronchi, thoracic duct, or diaphragm, but clearly these must be considered in assessing chest injuries.

Finally, I should remind you that in children the thoracic cage is so elastic that it is possible for severe compression of the chest contents to take place, without much in the way of external evidence of damage to the chest wall.

\title{
REFERENCES
}

Cheshire, D. J. E. \& Coats (1966). Int. F. Paraplegia, 4, I.

Clarke, R., Topley, E. \& Flean, C. T. G. (1955). Lancet, i, 629.

Clarke, R. \& Fisher, M. R. (1956). Br. F. clin. Pract. I o, 746.

\section{FREQUENCY AND DISTRIBUTION OF ASSOCIATED INJURIES IN TRAUMATIC PARAPLEGIA AND TETRAPLEGIA}

\author{
By F.-W. MeInecke, M.D. \\ From the Spinal Centre of the Surgical Department of the Berufsgenossenschaftlichen \\ Krankenanstalten Bergmannsheil, Bochum, Germany
}

\section{INTRODUCTION}

TRAUMATIC paraplegia usually impresses laymen and doctors to a point where their whole attention is focussed on this condition. For the injured it means in most cases an acute danger of life which continues to exist for a shorter or longer span of time. The prominence of this condition quite often determines all further measures and may lead to neglecting or overlooking associated injuries. It is these associated injuries, however, which can be of great consequence, as they increase the danger to life. The lack of sensation due to paralysis often obscures the extent of the total injury and thus renders diagnosis more difficult. Also, paraplegia might require different methods in the treatment of the associated injuries than those normally applied in patients without injuries of the spinal cord. It is the subject of this paper to examine the following questions:

I. Is paraplegia often associated with other injuries?

2. Are there typical accidents resulting in paraplegia?

3. Are there typical associated injuries in specific accidents?

4. Are there types of associated injuries which occur with particular frequency to different parts of the body?

We shall not discuss any specific methods of treatment as this would exceed the subject of this paper. 